\title{
Exploring the potential of variola virus infection of cynomolgus macaques as a model for human smallpox
}

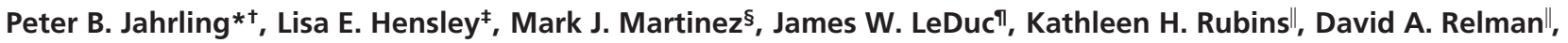 \\ and John W. Huggins ${ }^{\ddagger}$
}

\begin{abstract}
*Headquarters and ₹Virology and \$Pathology Divisions, U.S. Army Research Institute of Infectious Diseases, Frederick, MD 21702; ${ }^{1}$ National Center for Infectious Diseases, Centers for Disease Control and Prevention, Atlanta, GA 30333; and IDepartment of Microbiology and Immunology, Stanford University, Stanford, CA 94305
\end{abstract}

Communicated by Patrick O. Brown, Stanford University School of Medicine, Stanford, CA, August 12, 2004 (received for review May 29, 2004)

\begin{abstract}
Smallpox virus (variola) poses a significant threat as an agent of bioterrorism. To mitigate this risk, antiviral drugs and an improved vaccine are urgently needed. Satisfactory demonstration of protective efficacy against authentic variola will require development of an animal model in which variola produces a disease course with features consistent with human smallpox. Toward this end, cynomolgus macaques were exposed to several variola strains through aerosol and/or i.v. routes. Two strains, Harper and India 7124, produced uniform acute lethality when inoculated i.v. in high doses $\left(10^{9}\right.$ plaque-forming units). Lower doses resulted in less fulminant, systemic disease and lower mortality. Animals that died had profound leukocytosis, thrombocytopenia, and elevated serum creatinine levels. After inoculation, variola was disseminated by means of a monocytic cell-associated viremia. Distribution of viral antigens by immunohistochemistry correlated with the presence of replicating viral particles demonstrated by electron microscopy and pathology in the lymphoid tissues, skin, oral mucosa, gastrointestinal tract, reproductive system, and liver. These particles resembled those seen in human smallpox. High viral burdens in target tissues were associated with organ dysfunction and multisystem failure. Evidence of coagulation cascade activation (D dimers) corroborated histologic evidence of hemorrhagic diathesis. Depletion of T cell-dependent areas of lymphoid tissues occurred, probably as a consequence of bystander apoptotic mechanisms initiated by infected macrophages. Elaboration of cytokines, including IL- 6 and IFN- $\gamma$, contribute to a cytokine storm formerly known as "toxemia." A more precise understanding of disease pathogenesis should provide targets for therapeutic intervention, to be used alone or in combination with inhibitors of variola virus replication.
\end{abstract}

$\mathrm{s}$ mallpox is widely regarded as a significant bioterrorist threat (1). Ironically, an unanticipated consequence of the World Health Organization (WHO) global smallpox eradication campaign (2) was the creation of a largely susceptible population; in the United States, routine smallpox vaccination was discontinued in 1976. To mitigate a smallpox attack, serious consideration is being given to vaccinating large segments of the population; however, conventional vaccination carries a significant adverse reaction rate, and thus a number of population segments are excluded. The need for a protective but less reactogenic vaccine is apparent, and the availability of an antiviral drug to treat those exposed to the smallpox virus (variola) would be highly desirable.

To address these research needs, work with this virus was recommended by a U.S. Institute of Medicine study group in 1999 (3), and a research plan was approved by the WHO in accordance with WHO Resolution 52.10 (4) to develop an animal model by using authentic variola virus. The rationale for this initiative was to fulfill the requirements of the U.S. Food and Drug Administration (FDA) animal efficacy rule (5), which was established to facilitate the approval of therapeutic strategies and vaccines for microbial agents where it is impractical or unethical to obtain efficacy data in humans. In the case of smallpox virus, which no longer occurs in human populations, the only option for demonstrating protective efficacy is in an animal model. The FDA animal efficacy rule requires that the authentic agent (in this case, variola) be used and that the disease process be faithful to the human disease. The study endpoint must be based on reduced morbidity or mortality. For evaluating countermeasures against smallpox, adherence to these requirements is especially challenging. There are no animal reservoirs for variola virus in nature, and most animal species cannot even be infected in the laboratory (2). Attempts to develop nonhuman primate models for variola infection and disease in the 1960s met with only limited success $(6,7)$. However, the modified vaccinia Ankara strain was licensed at that time for use in Germany on the basis of reduction of symptomatology in primates exposed to variola (8). Encouraged by these pioneering studies, our interagency team (9) sought to establish a lethal model for authentic smallpox by exposing cynomolgus macaques to a number of variola strains in high doses, by a variety of routes. The initial constraint established by WHO Resolution 52.10 was that this work be completed before December 2002. In preliminary communications $(9,10)$, we reported the results of exposing two groups of monkeys to the Yamada and Lee strains administered as a fine-particle aerosol (analogous to a biological warfare attack). None of the monkeys exposed to the highest achievable aerosol dose $\left[10^{8.5}\right.$ plaque-forming units (pfu)] of either strain developed serious disease, although all exposed animals did become infected. With the December 2002 WHO deadline rapidly approaching, our first priority was to establish systemic disease; thus, we selected two additional variola strains (Harper and India 7124) and exposed monkeys to both aerosolized virus $\left(10^{8} \mathrm{pfu}\right)$ and i.v. inoculation $\left(10^{9} \mathrm{pfu}\right)$ to maximize the likelihood of producing serious, systemic disease with features of human smallpox. We achieved this goal, although further developmental work is required to model the full spectrum of human disease more faithfully. These initial trials, as well as subsequent developmental studies using the i.v. route alone, are the subject of this report.

\section{Materials and Methods}

Exposure of Monkeys to Variola. Thirty-six cynomolgus monkeys (Macaca fascicularis) were exposed to one of two variola strains,

Freely available online through the PNAS open access option.

Abbreviations: WHO, World Health Organization; FDA, U.S. Food and Drug Administration; pfu, plaque-forming unit.

See Commentary on page 14994.

†To whom correspondence should be addressed at: Headquarters, U.S. Army Research Institute of Infectious Diseases, 1425 Porter Street, Frederick, MD 21702-5011. E-mail: peter.jahrling@us.army.mil.

๑ 2004 by The National Academy of Sciences of the USA 
Table 1. Distribution of mortality in cynomolgus macaques exposed to variola strains

\begin{tabular}{|c|c|c|c|c|c|}
\hline Animal ID & Strain & Route & Dose, pfu & Death/total & Days of death \\
\hline$H-1,-2,-3,-4$ & Harper & i.v. plus aerosol & $10^{9}$ & $3 / 4$ & $4,4,6$ \\
\hline$H-5,-6,-7$ & Harper & i.v. & $10^{9}$ & $3 / 3$ & $4,6,10$ \\
\hline$H-8,-9,-10$ & Harper & i.v. & $10^{8}$ & $1 / 3$ & 11 \\
\hline $\mathrm{H}-11$ to -17 & Harper & i.v. & $10^{9}$ & $4 / 4$ & $4,4,5,4$ \\
\hline $\mid-1,-2,-3,-4$ & India & i.v. plus aerosol & $10^{9}$ & $4 / 4$ & $3,3,4,13$ \\
\hline $\mathrm{I}-5$ to -10 & India & i.v. & $10^{9}$ & $5 / 6$ & $3,4,6,4,10$ \\
\hline $\mid-11,-12,-13$ & India & i.v. & $10^{8}$ & $0 / 3$ & \\
\hline $\mathrm{I}-14,-15,-16$ & India & i.v. & $10^{7}$ & $0 / 3$ & \\
\hline $\mathrm{I}-17,-18,-19$ & India & i.v. & $10^{6}$ & $0 / 3$ & \\
\hline
\end{tabular}

Harper and India 7124. Before enrollment, all animals were clinically healthy and had negative test results for simian retrovirus, simian $T$ cell leukemia virus, simian immunodeficiency virus, and antibodies against vaccinia virus; both variola inoculum seeds were prepared as third-passage materials from chorioallantoic membranes in BSC-40 cells and were processed as described in Supporting Text, which is published as supporting information on the PNAS web site. Aerosols contained 1- to 3- $\mu \mathrm{m}$ particles as described for monkeypox virus (11). The use of i.v. exposures was through the femoral vein in $1.0-\mathrm{ml}$ volumes. Eight animals were challenged by a combination of aerosol and i.v. inoculation, four with the Harper strain and four with the India strain. Additional animals were exposed i.v. to virus alone in various doses (Table 1). Animals were examined daily after exposure. During the study, the animals were sedated with a tiletamine-zolazepam combination $(3-6 \mathrm{mg} / \mathrm{kg}$, i.m. with Telazol, A.H. Robbins, Richmond, VA) and bled at 2- to 3-day intervals for hematology, clinical chemistry, serology, and virus isolation. All animal experiments performed for this study adhered to National Institutes of Health guidelines.

Hematology, Coagulation Tests, and Biochemistry. Total white blood cell counts, white blood cell differential counts, red blood cell counts, platelet counts, hematocrits, total hemoglobin, mean cell volume, mean corpuscular volume, and mean corpuscular hemoglobin concentrations were determined from blood samples collected in EDTA by using an Act 10 Coulter Counter (Coulter Electronics, Hialeah, FL). Serum chemistries were determined by using a Piccolo point-of-care blood analyzer (Abaxis, Sunnyvale, CA). The level of D dimers (fibrin degradation products) was examined by using an ELISA according to the manufacturer's directions (Asserachrom D-Di, Diagnostica Stago, Parsippany, NJ).

Cytokine/Chemokine Production. IFN- $\alpha$, IFN- $\gamma$, tumor necrosis factor $\alpha$, IL-6, monocyte chemoattractant protein 1, macrophage inflammatory protein $1 \alpha$, and macrophage inflammatory protein $1 \beta$ levels in monkey sera/plasma were assayed by ELISA according to the manufacturer's directions (BioSource International, Camarillo, CA).

Necropsy. Complete necropsies were performed on all animals, either as soon as death occurred from infection or after humane killing of terminally ill or moribund animals by established guidelines. Animals that became clinically ill and subsequently recovered were killed and necropsied at 21 days postexposure. Tissues from all major organs were collected for histopathology and immunohistochemistry.

Histology, Immunohistochemistry, and Terminal Deoxynucleotidyltransferase-Mediated dUTP Nick End Labeling (TUNEL) Staining. Tissues, which were fixed by immersion in $10 \%$ neutral buffered formalin for a minimum of $48 \mathrm{~h}$, were subjected to $2 \times 10^{6}$ rads in a ${ }^{60} \mathrm{Co}$ irradiator to ensure viral inactivation. Viral antigen was immunohistochemically localized with a polyclonal antibody directed against vaccinia virus (kindly provided by B. Moss, National Institutes of Health, Bethesda) using previously described methods and formalin-fixed, paraffin-embedded tissues (12-14). Target cells were identified by a polyclonal vaccine antibody in conjunction with different cell markers. Antibodies to von Willebrand factor were used as endothelial cell markers, and a polyclonal macrophage marker, Ab-1 (Oncogene Research Products, San Diego), was used to identify monocytes/ macrophages. Apoptotic cells were detected by using a TUNEL assay (Apoptag, Intergen, Purchase, NY). Select target cells were identified by using a pan B cell marker, CD20 (DAKO), a pan $\mathrm{T}$ cell marker, CD3 (DAKO), a polyclonal macrophage marker, or an endothelial cell marker (von Willebrand factor, DAKO) in conjunction with the TUNEL assay. The presence of apoptotic cells in the tissues was confirmed by ultrastructural examination.

Ultrastructure. Tissues collected for ultrastructural examination were fixed in $2.5 \%$ glutaraldehyde on $0.1 \mathrm{M}$ phosphate buffer $(\mathrm{pH} 7.3)$, irradiated $\left(5 \times 10^{6}\right.$ rads $)$, and processed as described (14). Ultrathin sections were cut, placed on 200-mesh copper electron microscopy grids, stained with uranyl acetate and lead citrate, and examined by using a Philips transmission electron microscope.

Virus Isolation from Blood and Tissues. Infectious virus in tissues and circulating cells was assayed by counting plaques on Vero-E6 monolayers as described (15). Briefly, monolayers of Vero E6 cells were overlaid with serial dilutions of $10 \%$ organ homogenates or disrupted cell suspensions. After a 1-h absorption, the medium was replaced, and plates were incubated for 3-4 days and stained with $1.3 \mathrm{mg} / \mathrm{ml}$ crystal violet, $5 \%$ ethanol, and $30 \%$ formalin. After $20 \mathrm{~min}$, plates were washed and dried, and six plaques were counted.

\section{Results}

In contrast with previous experience (9), both variola strains produced rapidly fulminant disease, with almost uniform lethality and end-stage lesions resembling the human disease, in monkeys exposed by a combination of aerosol and i.v. routes. Three of four monkeys exposed to the Harper strain died (days 4, 4, and 6), and all four monkeys exposed to the India 7124 strain died (days 3, 3, 4, and 13) (see Table 2, which is published as supporting information on the PNAS web site). To determine whether i.v. inoculation alone was sufficient to produce lethality, we inoculated four more monkeys with the India strain $\left(10^{9} \mathrm{pfu}\right.$ i.v.) and obtained uniform lethality (days 3, 3, 4, and 6). Thus, we demonstrated the fundamental observation that it is possible to achieve lethal infection of primates by using variola virus. To determine whether lower doses of virus produced a less fulminant disease course, we titrated the India strain for lethality in 

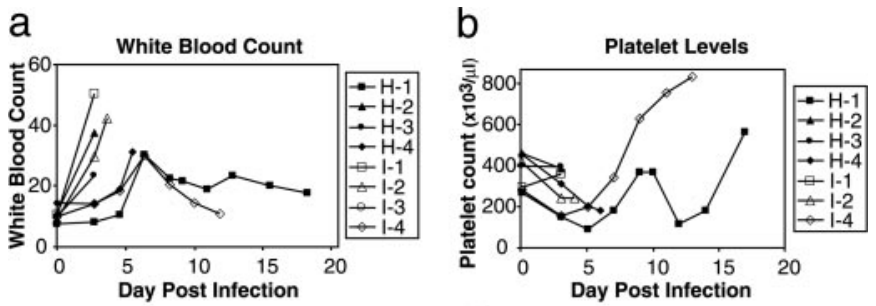

C
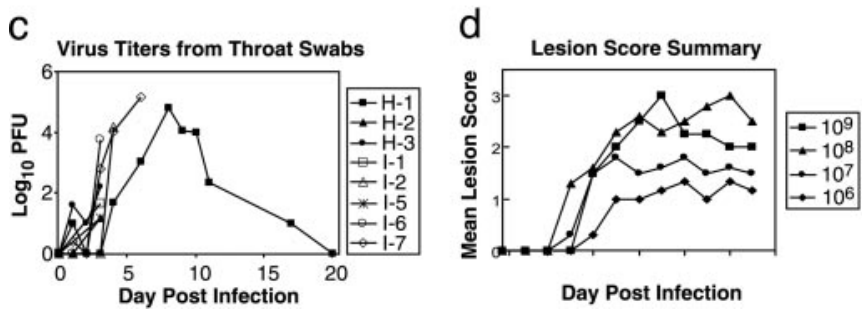

Fig. 1. Total white blood counts in monkeys infected with Harper and India strains of smallpox (a), platelet counts in plasma from infected monkeys (b), infectious viral titers recovered from throat swabs of infected monkeys (c), and skin lesions scores as a function of viral dose (India strain) (d). Lesion scores: 3 , $>500$ lesions; $2,50-500$ lesions; 1, 1-50 lesions.

monkeys by exposing animals i.v. to various 10 -fold dilutions. In this series, only one of two monkeys receiving $10^{9} \mathrm{pfu}$ died; none of those receiving $10^{8}$ pfu or lower doses died, although the severity of objective markers of disease did decline in a doserelated manner (see below). Likewise, the Harper strain was uniformly lethal at $10^{9}$ pfu i.v., but only one of three animals died at a 10 -fold lower dose (Table 1).

Total peripheral white blood counts were markedly elevated in monkeys dying acutely; differential counts revealed a monocytosis $>20 \%$ in those that died before day 6 (Fig. 1a). Leukocytosis peaked later in monkeys that lived longer. A transient decline in platelet counts was observed in all animals acutely infected, although rarely to levels $<100,000$ per microliter (Fig. 1b). Infectious virus was isolated from peripheral blood monocytes within 2 days of inoculation, although never in cell-free plasma. Titers ranged from $10^{2}$ to $10^{6}$ pfu per $10^{6}$ cells and increased with time. Infectious virus also was readily isolated from oropharyngeal swabs (Fig. 1c). Whereas swabs obtained early after aerosol exposure might have included inoculum virus, titers after day 3 likely represented progeny, replicating virus in the pharynx, as did titers from animals inoculated i.v. at even earlier time points.

Clinically, these monkeys showed cutaneous erythema and dermal hemorrhage as early as day 2 . By day 3 , early development of vesicles and pustules were evident. All animals developed fevers within 3 days, followed by anorexia, cough, and skin lesions, characterized by centrifugal distribution with concentration on the extremities. Many of these features resembled those described in humans in the past. The numbers of skin lesions varied with the dose. Animals that were exposed to the highest viral doses $\left(10^{9}\right.$ and $\left.10^{8} \mathrm{pfu}\right)$ and that lived past day 7 consistently developed $>500$ lesions (Fig. 1d). At necropsy, in addition to cutaneous hemorrhage, features of a coagulopathy were observed, which included hemorrhagic effusions in the thoracic and abdominal cavities and the ocular anterior chamber, in addition to visceral and mucosal hemorrhage.

Clinical chemistry values for monkeys inoculated with variola Harper or India strains are tabulated by days for individual animals (Tables 3 and 4, which are published as supporting information on the PNAS web site). Serum transaminase levels were markedly elevated by day 4 in monkeys exposed to the India strain; blood urea nitrogen and creatinine were likewise markedly elevated by times proximate to death. A steady decline in

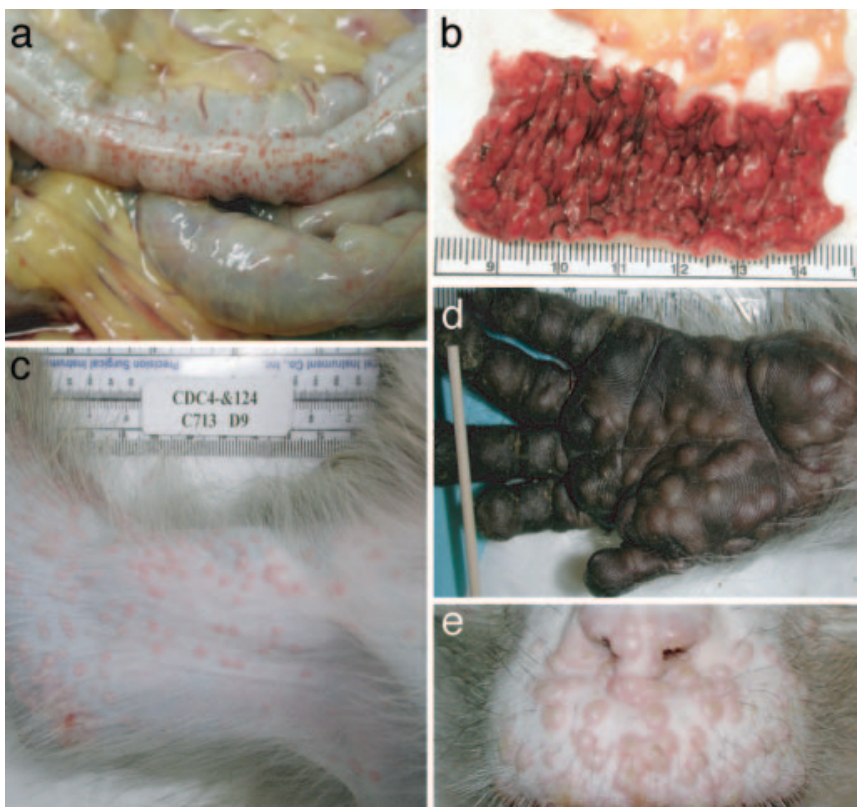

Fig. 2. Gross lesions associated with smallpox virus infection of monkeys. (a) Hemorrhage on the serosal surface of the distal colon, monkey I-7, 6 days after exposure. Note the diffuse petechial hemorrhages and hemorrhagic, colic lymph nodes. (b) Mucosal surface of the distal colon described in a. Note the severe congestion, hemorrhage, and hemorrhagic colic lymph nodes. (c) Medial surface of the right arm of monkey I-4, 9 days after exposure. Smallpox pustules are predominantly discrete with occasional coalescence. Pock lesions developed synchronously and were more numerous distally, which is consistent with centrifugal distribution. (d). Palmar surface of the left hand of a monkey 11 days after exposure. Discrete and coalescing pustules retained integrity due to heavily keratinized palmar and digital epidermis. (e) Upper lip and nostrils from a cynomolgus monkey 11 days after exposure. Note synchronous development of pustules, some of which have umbilicated. Pustules that formed on the lightly keratinized mucosal surfaces of the nostrils have already ulcerated, resulting in dried exudates.

serum albumin, with stable total protein values, suggested a capillary leak syndrome.

Postmortem examinations were performed on all animals. Evaluation of infectious viral burdens in organs of monkeys at necropsy revealed viral titers in vast excess of the inoculum, ranging from $10^{5} \mathrm{pfu} / \mathrm{g}$ of brain to $>10^{9} \mathrm{pfu} / \mathrm{g}$ in adrenal, kidney, spleen, and liver. A detailed report on the pathological findings will be submitted separately (M.J.M., L.E.H., J.W.H., W. Shieh, S. Zaki, and P.B.J., unpublished work). In brief, many organs displayed evidence of diffuse petechial hemorrhages on serosal surfaces (Fig. 2a) and severe congestion and hemorrhage on mucosal surfaces, often in association with hemorrhagic, colic lymph nodes (Fig. 2b). Vesicular and pustular lesions erupted synchronously in a centrifugal distribution, more numerous on the extremities than on the trunk (Fig. $2 c$ and $d$ ). Pustules also developed on the face, and they eventually umbilicated and dried in animals that lived longer than 7 days (Fig. 2e).

At the microscopic level, lymphocyte depletion and hepatocellular and renal tubular degeneration were consistent findings. Fibrin deposition and thrombi and systemic lymphocyte apoptosis involving secondary lymphoid organs also were present. Histopathologic examination of skin lesions revealed epithelial degeneration and necrosis, inter- and intracellular edema, and formation of microvesicles and micropustules, typical of human exanthema (Fig. 3a). Variola antigens were localized to Kupffer cells and hepatocytes in liver (Fig. $3 b$ ) and both proximal and distal tubules in kidney (Fig. $3 c$ ). Electron microscopy demonstrated replicating virus in most visceral tissues examined, 
a
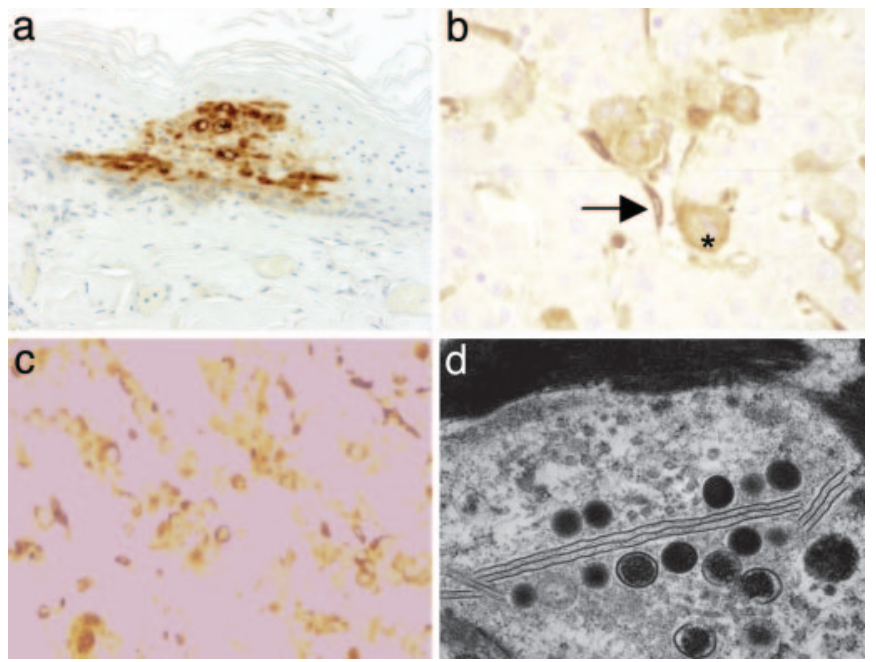

Fig. 3. Microscopic localization of smallpox virus in tissues of infected monkeys. (a) Immunohistochemical localization of variola antigen in cutaneous epidermis in association with hydropic degeneration and microvesicles, day 3. (b) Variola localization in hepatocytes and Kupffer (arrow) cells of liver, day 6. (c) Variola antigen in association with proximal and distal tubules of kidney, day 6. (d) Electron micrograph of histiocytic cells in spleen, with evidence of replicating virus in association with lamellar membranous bodies and intercellular fibrin.

especially lymph nodes and spleen, and fibrin deposition was frequently associated with infected macrophages in spleen (Fig. $3 d$ ). T lymphocyte-dependent areas of lymphoid tissues were generally depleted, whereas adjacent macrophages were productively infected.

Immunofluorescence examination of these tissues revealed variola antigens in association with monocytes/macrophages in
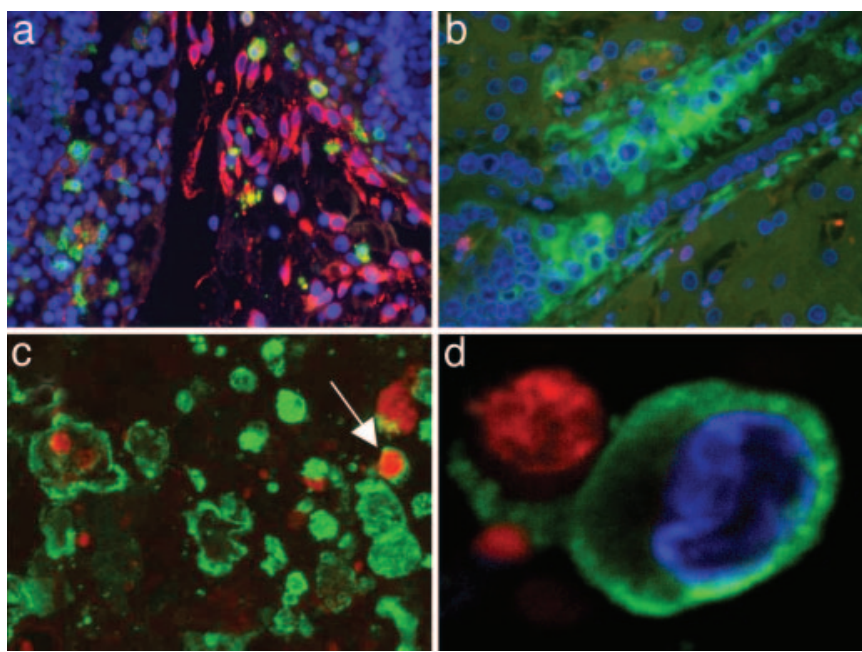

Fig. 4. Immunofluorescence examination of tissues from nonhuman primates infected with variola. (a) Staining for monocytes/macrophages (green) and viral antigen (red) indicated the presence of infected monocytes/ macrophages (gold) in the lymphoid tissues (lymph node shown) and in circulation. (b) In addition to monocytes/macrophages, virus-infected endothelial cells (green) were readily observed. (c) Staining for monocytes/ macrophages (green) and apoptosis (red) revealed the presence of numerous tingible body macrophages in lymphoid tissues. The majority of apoptotic cells were not monocytes/macrophages but instead were lymphocytes. A rare apoptotic monocyte/macrophage (arrow) is shown. (d) A monocyte/ macrophage with a clearly stained nonapoptotic nucleus (blue) is shown engulfing two separate apototic bodies. a

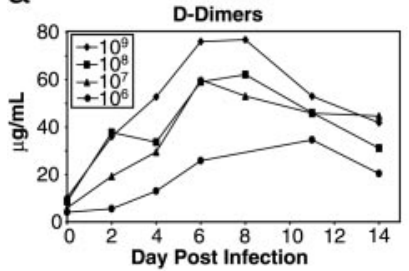

b

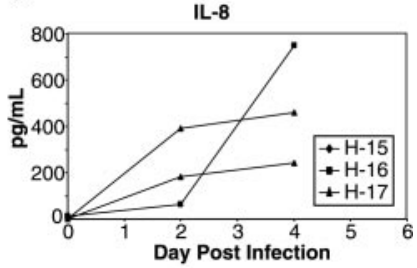

C

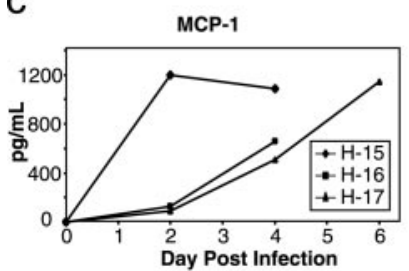

e

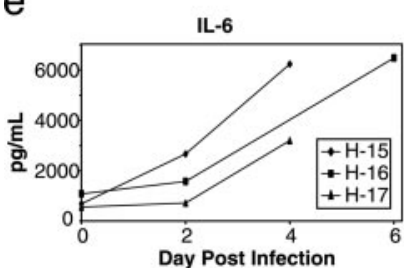

d

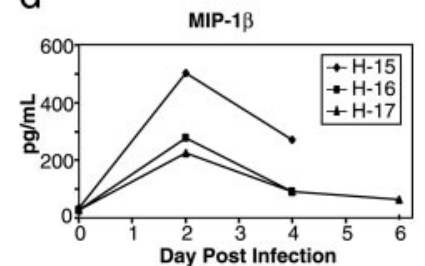

f

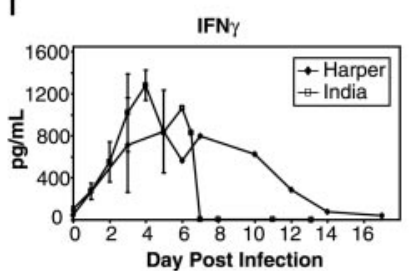

Fig. 5. Concentrations of $\mathrm{D}$ dimers, cytokines, and chemokines sequentially obtained from variola-infected monkeys. (a) D dimers $(\mu \mathrm{g} / \mathrm{ml})$ in sera of monkeys that received graded doses of variola strain India 1724 i.v. (b) IL-8 concentrations (pg/ml). (c) Monocyte chemoattractant protein 1 concentrations $(\mathrm{pg} / \mathrm{ml})$. ( $d$ ) Macrophage inflammatory protein $1 \beta$ concentrations (pg/ $\mathrm{ml})$. (e) IL-6 concentrations ( $\mathrm{pg} / \mathrm{ml}$ ) in the sera of three monkeys infected i.v. with Harper strain ( $\left.10^{9} \mathrm{pfu}\right)$. ( $f$ ) Mean ( $\pm 1 \mathrm{SD}$ ) IFN- $\gamma$ concentrations ( $\mathrm{pg} / \mathrm{ml}$ ) in sera of monkeys infected i.v. with India 7124 and Harper strains (10 $10^{9} \mathrm{pfu}$ ).

the lymphoid tissues (Fig. $4 a$ ) and in the circulation. In addition to monocytes/macrophages, virus-infected endothelial cells were readily observed (Fig. 4b). Staining for monocytes/ macrophages (green) and apoptosis (red) revealed the presence of numerous tingible body macrophages. The majority of apoptotic cells were not monocytes/macrophages but rather lymphocytes. A rare apoptotic monocyte/macrophage was visualized (Fig. 4c), and a monocyte/macrophage with a clearly stained nonapoptotic nucleus (blue) engulfing two separate apoptotic bodies was observed (Fig. $4 d$ ).

Consistent with a hemorrhagic diathesis, D-dimer values increased markedly in the sera of infected monkeys in correlation with viral inoculum size (Fig. 5a). Replication of variola in target tissues almost certainly resulted in organ failure by direct and indirect mechanisms. Viral replication in macrophages is often associated with initiation of a cytokine storm, which, once initiated, may cascade into a series of events largely independent of viral replication. To evaluate possible components of such a cascade, we measured values for cytokines in monkey sera by using immunoassays designed to detect human proteins but known to be cross reactive with those from macaques. Dramatic increases were observed for IL-8 in all lethally infected, variolainoculated monkeys examined (Fig. $5 b$ ). Similar increases were observed for monocyte chemoattractant protein 1, macrophage inflammatory protein $1 \beta$, IFN- $\gamma$, IL-6, and IFN- $\gamma$ (Fig. $5 c-f$ ).

\section{Discussion}

The demonstration that variola virus strains can produce lethal disease in monkeys is a significant advance toward the development of antiviral drugs and improved vaccines, as well as an improved understanding of variola pathogenesis. The results will 
mitigate the threat of smallpox virus being reintroduced into an immunologically naïve human population. With additional refinement, this model should permit the demonstration of protective efficacy for such countermeasures in compliance with the FDA animal efficacy rule (5). The i.v. inoculation of high doses of variola virus produced an overwhelming, hemorrhagic disease course, ending in acute deaths, usually within 6 days of inoculation. Ideally, the model would include near uniform mortality, but a longer mean time to death, with evolution of disease more reminiscent of ordinary forms of smallpox rather than the hemorrhagic type. Our attempts to achieve this goal with a 10 -fold lower dose resulted in lower mortality (zero of three and one of three for the Harper and India strains, respectively). At present, the tradeoff is reduced mortality for reduced hemorrhage. Reduced mortality correlated with a dose-related reduction in clinical signs, including cutaneous and oral lesion counts and viral titers, objective parameters that might be accepted in lieu of mortality data in compliance with the FDA animal efficacy rule.

The use of i.v. inoculation of variola effectively eclipses the incubation and prodromal phases of naturally acquired smallpox infection in humans by creating instantaneous viremia and systemic spread of virus to target tissues. Yet, the sequence of events in the monkeys is similar to human hemorrhagic smallpox, a form that was almost always fatal (2). Use of this model for demonstrating antiviral drug efficacy sets the bar high; target organs are already seeded with virus, whereas in human smallpox, this evolution does not occur until 10 or more days after exposure (16). Thus, the temporal window of opportunity for effective intervention is very short. Model refinements worthy of further investigation include testing alternative routes of exposure by using the Harper and India strains, including mucosal and intratracheal instillation, although our initial studies using different variola strains by aerosol were unsuccessful (9). Future testing of the virulence of variola isolated from monkeys dying late (e.g., Monkey H-9), on the theory that selective pressures might have amplified a monkey-adapted variola strain from a heterogeneous viral inoculum, is warranted. Analysis of viral clones selected from vaccinia virus stocks recently revealed a

1. Henderson, D. A., Inglesby, T. V., Bartlett, J. G., Ascher, M. S., Eitzen, E., Jahrling, P. B., Hauer, J., Layton, M., McDade, J., Osterholm, M. T., et al. (1999) J. Am. Med. Assoc. 281, 2127-2137.

2. Fenner, F., Henderson, D. A., Arita, I., Jezek, Z. \& Ladnvi, I. D. (1988) in WHO Advisory Committee on Variola Virus Research (W.H.O., Geneva), pp. 296-309.

3. Institute of Medicine (1999) in Assessment of Future Scientific Needs for Live Variola Virus (Natl. Acad. Press, Washington, DC), pp. 81-85.

4. World Health Organization (1999) in WHO Advisory Committee on Variola Virus Research (W.H.O., Geneva).

5. 67 Federal Register 105 (2002), pp. 37988-37998.

6. Hahon, N. (1961) Bacteriol. Rev. 25, 459-476.

7. Hahon, N. \& Wilson, B. J. (1960) Am. J. Hyg. 71, 69-80.

8. Hochstein-Mintzel, V., Hanichen, T., Huber, H. C. \& Stickl, H. (1975) Zentralb. Bakteriol. Mikrobiol. Hyg. Ser. A 230, 283-297.

9. LeDuc, J. W. \& Jahrling, P. B. (2001) Emerg. Infect. Dis. 7, 155-157.

10. Jahrling, P., Zaucha, G. \& Huggins, J. (2000) in Emerging Infections (Am. Soc. Microbiol., Washington, DC), Vol. 4, pp. 187-200.

11. Zaucha, G. M., Jahrling, P. B., Geisbert, T. W., Swearengen, J. R. \& Hensley, L. (2001) Lab. Invest. 81, 1581-1600. spectrum of virulence phenotypes (17). Monkey-adapted variola virus might produce systemic infections by more natural routes of exposure and at lower viral doses.

Despite its limitations, the i.v. variola primate model as described has already provided valuable insight into the pathogenesis of this exquisitely adapted human pathogen. After i.v. inoculation, variola virus disseminated via a monocytic cellassociated viremia, resulting in lesions affecting the lymphoid tissues, skin, oral mucosa, gastrointestinal tract, reproductive system, kidney, and liver. High viral burdens and associated degenerative changes in target tissues contribute to organ dysfunction and multisystem failure. Evidence of coagulation cascade activation ( $\mathrm{D}$ dimers) and declines in platelets corroborate histologic evidence of hemorrhagic diathesis. Depletion of $\mathrm{T}$ cell-dependent areas of lymphoid tissues occurs that is probably a consequence of bystander apoptotic mechanisms initiated by infected macrophages (18).

Elaboration of cytokines including IL-6, IL-8, and INF- $\gamma$ contributes to the cytokine storm formerly known as "toxemia." A more precise understanding of these mediators should provide targets for therapeutic intervention, to be used alone or in combination with inhibitors of variola virus replication. Genome-wide host transcriptional analysis in these same monkeys has revealed additional insights (18).

Variola and monkeypox viruses produce very similar diseases in monkeys and resemble smallpox in humans, albeit with an accelerated disease course. By parallel development of both the variola and monkeypox primate models, we expect to establish links between them to facilitate all but the most critical testing of candidate countermeasures in the monkeypox model, as was reported recently for an alternative smallpox vaccine (modified vaccinia Ankara) (19). The variola primate model would be reserved for testing only those countermeasures that have passed all other FDA requirements for drug or vaccine licensure.

We thank the members of the laboratories of Drs. Inger Damon, J.W.H., Louise Pitt, Sherif Zaki, and Tom Ksiazek, as well as the veterinary medicine departments of the U.S. Army Research Institute of Infectious Diseases and Centers for Disease Control and Prevention for their invaluable contributions to this work.

12. Geisbert, T. W., Hensley, L. E., Gibb, T. R., Steele, K. E., Jaax, N. K. \& Jahrling, P. B. (2000) Lab. Invest. 80, 171-186.

13. Schoepp, R. J., Morin, M. D., Martinez, M. J., Kulesh, D. A., Hensley, L. Geisbert, T. W., Brady, D. R. \& Jahrling, P. B. (2003) Lab. Invest. 84, 41-48.

14. Geisbert, T. W., Young, H. A., Jahrling, P. B., Davis, K. J., Larsen, T., Kagan, E. \& Hensley, L. E. (2003) Am. J. Pathol. 163, 2371-2382.

15. Martinez, M. J., Bray, M. P. \& Huggins, J. W. (2000) Arch. Pathol. Lab. Med. 124, 362-377.

16. Breman, J. G. \& Henderson, D. A. (2002) N. Engl. J. Med. 346, 1300 1308.

17. Weltzin, R., Liu, J., Pugachev, K. V., Myers, G. A., Coughlin, B., Blum, P. S., Nichols, R., Johnson, C., Cruz, J., Kennedy, J. S., et al. (2003) Nat. Med. 9, 1125-1130.

18. Rubins, K. H., Hensley, L. E., Jahrling, P. B., Whitney, A. R., Geisbert, T. W., Huggins, J. W., Owen, A., LeDuc, J. W., Brown, P. O. \& Relman, D. A. (2004) Proc. Natl. Acad. Sci. USA 101, 15190-15195.

19. Earl, P. L., Americo, J. L., Wyatt, L. S., Eller, L. A., Whitbeck, J. C., Cohen, G. H., Eisenberg, R. J., Hartmann, C. J., Jackson, D. L., Kulesh, D. A., et al. (2004) Nature 428, 182-185. 